\title{
Melanoma Advanced Imaging Dermatoscope
}

National Cancer Institute

\section{Source}

National Cancer Institute. Melanoma Advanced Imaging Dermatoscope. NCI Thesaurus.

Code C154588.

A dermatoscope designed to use hyperspectral imaging and software algorithms to identify early-stage skin melanomas with high specificity. 